\title{
CHEMICAL ABUNDANCES IN GALACTIC BULGE PN
}

\author{
N.A. WALTON and M.J. BARLOW \\ Department of Physics \& Astronomy, University College London, \\ Gower Street, London WC1E 6BT, England
}

and

R.E.S. CLEGG

Royal Greenwich Observatory, Madingley Road, Cambridge CB3 OEZ, England

\begin{abstract}
We present abundance determinations, in particular of carbon, and C/O ratios, for 11 Galactic bulge planetary nebulae (PN) based on our low resolution UV data from IUE observations and optical spectrophotometry from the Anglo-Australian Telescope. We compare the observed abundances with those predicted by dredge-up theory for the high metallicity Galactic bulge. The sample abundances are also contrasted with the abundances found for PN in the Galactic disk. The mean $\mathrm{C} / \mathrm{O}$ ratio for the bulge PN is significantly lower than that found for Galactic disk PN. Further, we present an abundance analysis of the very metal-poor bulge PN M2-29. From an analysis of the differential extinction found from the observed ratios of the He II $1640,4686 \AA$ lines, we find that the ultraviolet reddening law towards the bulge is steeper than in the solar neighbourhood.
\end{abstract}

Table: Our abundances determined for the bulge PN and the values found for local neighbourhood PN, taken from Torres-Peimbert \& Peimbert (1977, Rev Mex Astron Astrofis, 2, 181) and Aller \& Czyzak (1983, ApJS, 51, 211), are shown.

M2-29 is excluded from the means as it is a low metallicity halo PN located in the bulge.

* The only one of our bulge PN with $\mathrm{C} / \mathrm{O}$ clearly in excess of unity (Cn 1-5) has a WC4 Wolf-Rayet central star.

\section{Bulge PN Elemental Abundances}

$\begin{array}{llllllll}\text { Object } & \mathrm{He} & \mathrm{C} & \mathrm{N} & \mathrm{O} & \mathrm{Ne} \\ & & \times 104 & \times 104 & \mathrm{C} / \mathrm{O} & \mathrm{N} / \mathrm{O} \\ & & & & & & \\ \text { M 3-21 } & 0.118 & 2.28 & 2.98 & 7.04 & 1.57 & 0.32 & 0.42 \\ \text { H 1-42 } & 0.107 & - & 0.97 & 5.34 & 0.74 & - & 0.18 \\ \text { M 2-23 } & 0.084 & - & 1.34 & 2.93 & 0.45 & - & 0.46 \\ \text { M 1-42 } & 0.194 & 0.72 & 3.21 & 1.94 & 0.63 & 0.37 & 1.65 \\ \text { Cn 1-5* } & 0.143 & 13.3 & 3.98 & 7.67 & 2.44 & 1.74 & 0.52 \\ \text { M 2-30 } & 0.112 & 0.99 & 1.08 & 4.65 & 1.05 & 0.21 & 0.23 \\ \text { Hb 8 } & 0.104 & 0.35 & 0.32 & 3.29 & 0.60 & 0.11 & 0.096 \\ \text { Al 1 } & 0.103 & 5.36 & 2.31 & 4.75 & 1.03 & 1.13 & 0.49 \\ \text { Vy 2-1 } & 0.105 & 4.35 & 1.94 & 5.96 & 1.38 & 0.73 & 0.33 \\ \text { M 3-33 } & 0.112 & 0.55 & - & 2.61 & - & 0.21 & - \\ \text { M 2-29 } & 0.093 & 0.01 & 0.17 & 0.28 & 0.039 & 0.036 & 0.62 \\ \text { Local PN } & 0.100 & 5.50 & 0.91 & 4.79 & 0.96 & 1.15 & 0.19 \\ \text { Bulge mean } & 0.118 & 3.49 & 2.01 & 4.62 & 1.10 & 0.60 & 0.49 \\ \quad \text { 士 } & 0.029 & 4.10 & 1.14 & 1.83 & 0.59 & 0.53 & 0.44\end{array}$

